\section{Facharztprüfung zur Erlangung des Facharzttitels FMH für Kinder- und Jugendmedizin}

Aufgrund des Weiterbildungsprogrammes, welches am 1. Juli 2001 in Kraft gesetzt wurde, ist die Teilnahme an der Facharztprüfung für diejenigen Kandidatinnen und Kandidaten Voraussetzung für die Erlangung des Facharzttitels FMH für Kinderund Jugendmedizin, welche ihre Weiterbildung in Kinder- und Jugendmedizin bis am 31. Dezember 1997 nicht abgeschlossen hatten. Es empfiehlt sich, die Facharztprüfung frühestens im letzten Jahr der reglementarischen Weiterbildung abzulegen (Art. 23 WBO).
Ort und Datum: Die Prüfung erfolgt in zwei Teilen:

- Praktische Prüfung: Oktober - Dezember 2002 (Datum und Ort werden bis Ende Juli 2002 mitgeteilt).

- Schriftliche Prüfung: 13. Dezember 2002, 9.00-13.00 Uhr, (Auditorium Ettore Rossi, Universitäts-Kinderklinik, Inselspital Bern).

Prüfungsgebühr: Die SGP erhebt eine Prüfungsgebühr von Fr. 650.- für die praktische Prüfung, Fr. 150.- für die schriftliche Prüfung.

Anmeldung: Anmeldeformulare können bezogen werden bei: SGP, Union patronale du canton de Fribourg, Rue de l'Hôpital 15, 1700 Fribourg, Tel. 02635033 44, Fax 02635033 04, E-Mail: pa.minder@unionpatronale.ch.

Anmeldefrist: 31. Mai 2002

\section{Facharztprüfung zur Erlangung des Facharzttitels FMH für Tropen- und Reisemedizin}

Aufgrund des Weiterbildungsprogrammes und des Beschlusses des Zentralvorstandes der FMH vom 28. Dezember 2000 ist das Bestehen der Facharztprüfung für alle Kandidatinnen und Kandidaten, welche ihre Weiterbildung nach dem 31. Dezember 2002 abschliessen, Voraussetzung zur Erlangung des Facharzttitels FMH für Tropen- und Reisemedizin. Näheres zu den Übergangsbestimmungen und Ausnahmen ist in der Schweizerischen Ärztezeitung Nr. 5 vom 30. Januar 2002 publiziert.
Ort: Schweizerisches Tropeninstitut, Basel

Zeitpunkt: Donnerstag, 7. November 2002

Prüfungsgebühr: Die Fachgesellschaft erhebt einen Unkostenbeitrag von Fr. 500.-.

Anmeldung/Information: Frau Beatrice Wäckerlin, Sekretariat der Schweiz. Fachgesellschaft für Tropenund Reisemedizin FMH, c/o Schweiz. Tropeninstitut, Postfach, 4002 Basel, Tel. 06128482 55, Fax 061 28481 83, E-Mail: tropmed@keep.touch.ch.

Anmeldefrist: 31. August 2002 


\section{Examen de spécialiste en vue de l'obtention du titre de spécialiste FMH en pédiatrie}

Conformément au programme de formation postgraduée entré en vigueur le $1^{\mathrm{er}}$ juillet 2001 , la participation à l'examen de spécialiste est une condition requise pour les candidats à l'obtention du titre de spécialiste FMH en pédiatrie qui n'avaient pas encore terminé leur formation postgraduée en pédiatrie le 31 décembre 1997. Il leur est recommandé de passer l'examen de spécialiste au plus tôt durant la dernière année de leur formation postgraduée réglementaire (art. $23 \mathrm{RFP}$ ).
Lieu et date: L'examen a lieu en deux parties:

- Examen pratique: octobre à décembre 2002 (lieux et dates exacts seront communiqués d'ici à fin juillet 2002).

- Examen écrit: le 13 décembre 2002, 9.00-13.00 h (Auditorium Ettore Rossi, Clinique universitaire de pédiatrie, Hôpital de l'Ile, Berne).

Taxe d'examen: La SSP prélève une participation aux frais de Fr. 650.- pour l'examen pratique, Fr. 150.- pour l'examen écrit.

Inscription: Les formulaires d'inscription peuvent être obtenus auprès de la SSP, Union patronale du canton de Fribourg, Rue de l'Hôpital 15, 1700 Fribourg, tél 02635033 44, fax 02635033 04, e-mail: pa.minder@unionpatronale.ch.

Délai d'inscription: le 31 mai 2002

\section{Examen de spécialiste en vue de l'obtention du titre de spécialiste FMH en médecine tropicale et médecine des voyages}

Conformément au programme de formation postgraduée et à la décision du Comité central de la FMH du 28 décembre 2000, la réussite de l'examen de spécialiste est une condition requise pour les candidats au titre FMH en médecine tropicale et médecine des voyages qui termineront leur formation postgraduée après le 31 décembre 2002. Pour de plus amples renseignements concernant les dispositions transitoires et les dérogations, veuillez vous référer à la publication du Bulletin des médecins suisses no 5, du 30 janvier 2002.
Lieu: Institut Tropical Suisse, Bâle

Date: Jeudi, 7 novembre 2002

Taxe d'examen: La Société de discipline médicale prélève une participation aux frais de Fr. 500.-.

Inscription/Information: Mme Beatrice Wäckerlin, Secrétariat de la Société Suisse de médecine tropicale et médecine des voyages FMH, c/o Institut Tropical Suisse, Case postale, 4002 Bâle, tél. 06128482 55, fax 06128481 83, e-mail: tropmed@keep.touch.ch.

Délai d'inscription: 31 août 2002 